\title{
Effect of "Nourishing Yin and Qi, Promoting Blood Circulation and Detoxification" on Endoplasmic Reticulum Stress CHOP Apoptotic Bypass in Diabetic Atherosclerosis
}

\author{
Fu Xianzhao*, Li Chunyan, Huang Wenhua, Li Xingchan, Qiu Haixian, Huang Guangming, \\ Cao Qiuxia, Lu Mian
}

Clinic Medical College, Youjiang Medical National College, Baise, China

Email address:

1620100638@qq.com (Fu Xianzhao)

${ }^{*}$ Corresponding author

\section{To cite this article:}

Fu Xianzhao, Li Chunyan, Huang Wenhua, Li Xingchan, Qiu Haixian, Huang Guangming, Cao Qiuxia, Lu Mian. Effect of "Nourishing Yin and Qi, Promoting Blood Circulation and Detoxification" on Endoplasmic Reticulum Stress CHOP Apoptotic Bypass in Diabetic Atherosclerosis. American Journal of Clinical and Experimental Medicine. Vol. 7, No. 5, 2019, pp. 111-118.

doi: 10.11648/j.ajcem.20190705.12

Received: September 19, 2019; Accepted: September 28, 2019; Published: October 15, 2019

\begin{abstract}
To explore the mechanism of Huoxue Jiedu Jiangtang Recipe (HJJR) in alleviating endoplasmic reticulum stress (ERS) - inflammation, inhibiting ERS apoptotic bypass of CHOP and alleviating diabetic atherosclerosis (AS). Diabetic AS rats were duplicated by feeding of high lipid diet and intraperitoneal injection of streptozotocin. After diabetes modeled successfully, the rats were randomly divided into model group, low-dose HJJR group (HJJR ${ }_{1}$ ), high-dose HJJR group $\left(\mathrm{HJJR}_{2}\right)$ and western medicine group (Gliquidone+ Benazepril), and accepted corresponding drugs for 2 months respectively. The same batch of rats were taken as normal control group and fed with common diet. The levels of glycosylated hemoglobin $(\mathrm{GHb})$, blood lipid, fasting serum insulin (FINS) and insulin resistance index (IRI) were measured in each group. The contents of inflammatory factors TNF- $\alpha$ and IL- 6 were detected by immunohistochemistry. The mRNA transcription of GRP78 and caspase-12 were tested by reverse- transcription polymerase chain reaction (RT-PCR). The apoptotic level of aortic cells was checked by TUNEL. Compared with the model group, all drug groups could significantly reduce GHb, IRI, TG and LDL-C $(\mathrm{P}<0.05)$, increase FINS and HDL-C $(\mathrm{P}<0.05)$, down-regulate transcription of CHOP and GRP78 $(\mathrm{P}<0.05)$, reduce inflammatory factors TNF-a, IL-6, and decreased aortic apoptosis index (AI) $(\mathrm{P}<0.05)$. Compared with the western medicine group, the effect of HJJR2 group was more significant $(\mathrm{P}<0.05)$. HJJR could alleviate insulin resistance, correct lipid metabolism disorder, depress endoplasmic reticulum stress-induced inflammatory reaction, and inhibit endoplasmic reticulum stress-induced apoptotic bypass of CHOP in arterial cells. The therapeutic effect is dose dependent.
\end{abstract}

Keywords: Diabetes, Atherosclerosis, Endoplasmic Reticulum Stress,

CCAAT Enhance-Binding Protein Homologous Protein, Huoxue Jiedu Jiangtang Recipe

\section{Introduction}

At present, the incidence and prevalence of diabetes mellitus, impaired glucose tolerance (IGT) and impaired fasting blood glucose (IFG) are on the rise, and are obviously younger, which has brought serious harm to human health and social development [1]. Diabetes mellitus complicated with cardiovascular and cerebrovascular diseases is a common chronic complication. Atherosclerosis (AS) is the main cause of cardiovascular and cerebrovascular diseases, and diabetes mellitus is an independent risk factor. The incidence of cardiovascular and cerebrovascular diseases in diabetic patients is significantly higher than that in non-diabetic patients. The death rate caused by diabetic coronary heart disease accounts for about $80 \%$ of diabetic mortality, and the incidence of cerebrovascular diseases in diabetic patients is also significantly higher than that in non-diabetic patients [2]. Therefore, looking for the prevention and treatment of diabetic AS has become an important topic in the diabetes academic circles and academia of cardiovascular and cerebrovascular 
diseases. Simple western medicine treatment is difficult to prevent or delay the progress of diabetic AS, and has great side effects. According to the theory of traditional Chinese medicine (TCM), diabetes mellitus and its complicated AS are based on the pathogenesis of deficiency of both Qi and Yin, and implication of dryness and heat. Qi deficiency and inability to transfuse blood, can lead to blood circulation slowly and stasis. With the passing of time, stagnation of blood can produce heat, and dryness-heat in diabetes mellitus can refine fluid to produce phlegm, while heat and blood stasis can develop into toxins. As a result, blood stasis, phlegm turbidity, heat toxin depose and hold-up in the veins, accumulate plaques (that is, atherosclerotic plaques) [3]. Previous animal studies have found that Huoxue Jiedu Jiangtang Recipe (HJJR) can inhibit endoplasmic reticulum stress (ERS) cell apoptosis bypass of c-JUN $\mathrm{N}$ terminal kinases (c-JNK), decrease inflammatory response, reduce aortic cell apoptosis, and play protective effect on arteries [4]. CCAAT Enhance-Binding Protein Homologous Protein (CHOP) is a specific transcription factor of ERS, and activated c-JNK can phosphorylate and modify $\mathrm{CHOP}$, increase the apoptotic effect of each other by regulating the activity of CHOP [5]. This study will further explore the molecular mechanism of HJJR preventing and treating diabetic AS from another ERS apoptotic signal pathway

\section{Methods}

\subsection{Establishment of Models}

102 Male SD rats weighing 230 $\pm 20 \mathrm{~g}$ were selected from the Laboratory Animal Center of Guangxi Medical University, with certificate number SCXK (Gui) 2014-002. At room temperature of $20-25^{\circ} \mathrm{C}, 7$ days after feeding with common feed, 10 hours on an empty stomach, rats were injected streptozotoein (STZ) (Beijing Hua Yang Biotechnology Co., Ltd.) $50 \mathrm{mg} / \mathrm{kg}$ in abdomen once. After 72 hours, fasting blood glucose (FBG) was measured with blood glucose test paper (Wuhan Doctor De Bioengineering Co., Ltd.), and rats with $\mathrm{FBG} \geq 16.7 \mathrm{mmol} / \mathrm{L}$ two consecutive times were used for diabetic models. Then the rats were fed with high-fat feed (including ordinary feed $78.2 \%$, cholesterol $1.5 \%$, porcine bile salt $0.3 \%$, yolk powder $10 \%$, lard 10\%) (made in Jiangsu synergy Bioengineering Co., Ltd.) for 3 months. Another $25 \mathrm{SD}$ rats in the same batch were injected with the same amount of saline intraperitoneally and fed with common diet as normal group.

\subsection{Grouping and Administration}

The modeled rats were randomly divided into model group 25 , Gliquidone+benazepril group (western medicine group) 26, low dose HJJR group (HJJR1) 26, high dose HJJR group (HJJR2) 25, and began to administer drugs. HJJR (composed of Ginseng, Ophiopogon japonicus, Schisandra chinensis, Astragalus membranaceus, Cornus officinalis, Yam, Rehmannia glutinosa, Turtle shell, Peach kernel, Danpi, Rhubarb, Coptis chinensis, Salvia miltiorrhiza, purchased from the Affiliated Hospital of Youjiang National Medical College) were made into extract, $1 \mathrm{~g}$ is equivalent to $10 \mathrm{~g}$ of the original medicine. The rats in HJJR1 group and HJJR2 group were fed with HJJR suspension at a daily dose of 1 $\mathrm{g} / \mathrm{kg}$ and $1.5 \mathrm{~g} / \mathrm{kg}$ respectively. The western medicine group was fed with benazepril (Beijing Novartis Pharmaceutical Co., Ltd.) $2.50 \mathrm{mg} / \mathrm{kg}$ and Gliquidone (Beijing Wanhui Shuanghe Pharmaceutical Co., Ltd.) $7.50 \mathrm{mg} / \mathrm{kg}$ daily, which were prepared for same intragastric volume as HJJR group, and continued to feed high-fat diet for 2 months. The model group was fed with high-fat diet, while the normal group was fed with normal diet. The experimental operations were carried out in super-clean worktable.

\subsection{Sampling}

After 60 days medicine feeding, 10 hours fasting, rats were checked FBG, and then were intraperitoneally injected with $8 \%$ pentobarbital sodium $2 \mathrm{~mL} / \mathrm{kg}$ to anesthetize, subsequently $8 \mathrm{ml}$ of blood was collected from inferior vena cava by opening the abdomen from the center of the abdomen. Sera was separated by $2000 \mathrm{r} / \mathrm{min} 10 \mathrm{~min}$ centrifugation, and kept in $-20^{\circ} \mathrm{C}$ refrigerator for testing test index. At the same time, the aortas were dissected on ice, and the thoracic aorta segments were taken and divided into two parts, one part was immersed in $4 \%$ polyformaldehyde for immunohistochemistry and cell apoptosis experiments, the other was refrigerated at $-80^{\circ} \mathrm{C}$ for RT-PCR experiments.

\subsection{Detection of Experimental Indicators}

\subsubsection{Detection of Biochemical Indicators}

Fasting serum insulin (FINS) and glycosylated hemoglobin $(\mathrm{GHb})$ levels were measured by enzyme-linked immunosorbent assay (ELISA), which kits were purchased from Beijing Huayueyang Biological Technology Co., Ltd. The experimental procedures were carried out strictly according to the instructions and the contents were measured according to the standard curve. The insulin resistance index (IRI) was estimated according to the HOMA-IR $($ HOMA-IR $=$ FINS $\times$ FPG/22.5) proposed by Matthews. The contents of low density lipoprotein cholesterol (LDL-C), high density lipoprotein cholesterol (HDL-C) and triglyceride (TG) were determined by automatic biochemical analyzer.

\subsubsection{Detection of Apoptosis of Aortic Cells by TUNEL}

The TUNEL kit was purchased from Beijing Huayueyang Biotechnology Co., Ltd. The aortas were embedded in paraffin and sliced into $4 \mathrm{um}$ slices, and processed through dewaxing $\rightarrow$ rinsing $\rightarrow$ adding protease $\rightarrow$ room temperature $15 \mathrm{~min} \rightarrow$ Adding $3 \% \mathrm{H}_{2} \mathrm{O}_{2}$ to inactivate endogenous peroxidase $\rightarrow$ Room temperature $15 \mathrm{~min} \rightarrow$ Adding TUNEL reagent $\rightarrow$ Reaction at $37^{\circ} \mathrm{C}$ for $1 \mathrm{~h} \rightarrow$ Adding peroxidase $\rightarrow$ Adding diaminobenzidine $(\mathrm{DAB}) \rightarrow$ Room temperature 10 min $\rightarrow$ Dehydrating with ethanol step by step $\rightarrow$ Vitrifying with dimethylbenzeneSealing with resin, then observating under optical microscopy. The nucleus of normal cells were blue and the nucleus of apoptotic cells were brown. Five fields with the largest number of non-overlapping positive 
cells were selected to calculate the percentage of positive cells, namely cell apoptotic index (CAI).

\subsubsection{Immunohistochemical Detection of TNF- $\alpha$ and IL-6}

Rabbit TNF- $\alpha$ and IL-6 antibodis were purchased from Beijing Zhongshan Jinqiao Biotechnology Co., Ltd. The aortas fixed with $4 \%$ polyformaldehyde were embedded in paraffin, cut into 4um thin sections and dewaxed with xylene, and processed through stepwise concentration of ethanol dehydration $\rightarrow$ Restoring antigen $\rightarrow$ Inactivation of endogenous peroxidase $\rightarrow$ Dropping and discarding sealing fluid $\rightarrow$ Adding TNF- $\alpha$ and IL-6 antibodis respectively $\rightarrow$ Adding biotin-labeled second antibody streptavidinperoxidase complex $\rightarrow$ DAB dyeing $\rightarrow$ Redyeing with hematoxylin. The positive expression of TNF- $\alpha$ and IL-6 were brown. Under the microscope, five non-overlapping positive cells were selected to take pictures, and image-Pro Plus Version 6.0 image analysis software was used to detect the optical density of positive cells (IOD).

\subsubsection{Detection of Transcriptional Levels of GRP78 and CHOP Molecules in Aorta by Real-time PCR}

The internal reference gene and target gene primers were designed and synthesized by Shanghai Jikai Gene Company. The upstream primer of internal reference gene GAPDH is 5'-TTCAACGCAGTCAAGG-3', downstream primer is 5'-CTCAGCACCAGCATCACC-3', amplified product is $114 \mathrm{bp}$. Upstream primers of target gene GRP78 is 5'-CTTGGTATTGAAACTGTGGG-3', downstream 5'-TGTTACGGTGGGCTGATTAT-3', amplified product $117 \mathrm{bp}$; CHOP upstream 5'-TGCCTTTCGCCTTTGAGACAG-3', downstream 5'-GCTTTGGGAGGTGCTTGTGAC-3', amplified product $218 \mathrm{bp}$. The aortas were removed from the refrigerator at $-80^{\circ} \mathrm{C}$, and the total RNA were extracted, its integrity was detected with electrophoresis performed on agarose gel. Then the operation were carried out according to the instructions of the reverse transcription kit: $42^{\circ} \mathrm{C} 50 \mathrm{~min}, 95^{\circ} \mathrm{C} 5$ min, reversed transcription into the cDNA. the obtained cDNA were amplified into the target gene (according to the instructions of the PCR kit), steps and circulating parameters: Firstly, $96^{\circ} \mathrm{C}$ $4 \mathrm{~min}$, one cycle, then processing three-step reactions, $94^{\circ} \mathrm{C} 30$ second, $58^{\circ} \mathrm{C} 30$ second, $72^{\circ} \mathrm{C} 30$ second, 40 cycles. Recorded the number of cycles to reach the set threshold (CT). The specificity of primers was detected by dissolution curve, and with $2^{-\Delta \Delta}{ }^{\mathrm{Ct}}$ representing the expression of target gene, $\Delta \Delta \mathrm{Ct}=$ Experience group $\left(\mathrm{Ct}_{\text {target gene }}-\mathrm{Ct}_{\mathrm{GAPDH}}\right)$-control group $\left(\mathrm{Ct}_{\text {target }}\right.$ gene- $\left.\mathrm{Ct}_{\mathrm{GAPDH}}\right)$. Practically, the expression level of the control group was 1, and the ratio was the relative expression level of the experimental group.

\subsection{Statistical Processing}

All data were expressed with mean \pm standard deviation $(\bar{x} \pm S)$, and processed with SPSS 19.0 software. Intergroup differences were analyzed by one-way ANOVA. The data were tested for homogeneity of variance. If the variance were homogeneous, LSD statistical method were put to use, otherwise Tambanes T2 method were used. $\mathrm{P}<0.05$ was considered to indicate the statistically significant difference. The difference was statistically significant with $\mathrm{P}<0.05$.

\section{Result}

\subsection{Changes in Rats During the Experimental Process}

After successful modeling, rats had more urine, more water, more food intake, yellowish fur color, weight loss, slow reaction, slow action, and the experimental processed successfully.

\subsection{Effects of HJJR on GHb, FINS, IRI and Blood Lipid in Rats}

Compared with the normal control group, the contents of $\mathrm{GHb}, \mathrm{TG}, \mathrm{LDL}-\mathrm{C}$ and IRI in the model control group increased significantly $(\mathrm{P}<0.01)$, while the contents of FINS and HDL-C decreased significantly $(\mathrm{P}<0.01)$. Compared with the model group, in each administration group, the contents of GHb, IRI, TG and LDL-C decreased, while the contents of FINS and HDL-C increased $(\mathrm{P}<0.05)$. Compared with western medicine group, HJJR2 had more significant effect $(\mathrm{P}<0.05)$. The effect of HJJR was better with its increase dose, but there was no significant difference between HJJR groups. The results are shown in Table 1.

Table 1. Effects of HJJR on serum lipids, GHb, FINS and IRI in rats $(\bar{x} \pm S)$.

\begin{tabular}{|c|c|c|c|c|c|c|c|}
\hline Group & $\mathbf{n}$ & GHb (mmol/L) & LDL-C (mmol/L) & HDL-C (mmol/L) & TG (mmol/L) & FINS (mU/L) & IRI \\
\hline normal group & 25 & $6.43 \pm 1.16$ & $0.93 \pm 0.62$ & $0.98 \pm 0.08$ & $0.93 \pm 0.13$ & $20.89 \pm 1.41$ & $5.21 \pm 1.13$ \\
\hline model group & 25 & $21.87 \pm 4.52^{\mathrm{a}}$ & $2.21 \pm 0.81^{\mathrm{a}}$ & $0.51 \pm 0.07^{\mathrm{a}}$ & $2.41 \pm 0.26^{\mathrm{a}}$ & $15.89 \pm 4.89^{\mathrm{a}}$ & $17.88 \pm 2.15^{\mathrm{a}}$ \\
\hline $\mathrm{HJJR}_{1}$ group & 26 & $14.76 \pm 4.08^{\mathrm{ab}}$ & $1.81 \pm 0.24^{\mathrm{ab}}$ & $0.77 \pm 0.08^{\mathrm{ab}}$ & $1.85 \pm 0.32^{\mathrm{ab}}$ & $17.22 \pm 4.78^{\mathrm{ab}}$ & $7.91 \pm 1.16^{\mathrm{ab}}$ \\
\hline western medicine group & 26 & $15.76 \pm 4.51^{\mathrm{ab}}$ & $1.79 \pm 0.21^{\mathrm{ab}}$ & $0.74 \pm 0.09^{\mathrm{ab}}$ & $1.99 \pm 0.23^{\mathrm{ab}}$ & $16.88 \pm 4.77^{\mathrm{ab}}$ & $8.33 \pm 1.29^{\mathrm{ab}}$ \\
\hline
\end{tabular}

Notes: Compared with the normal group, ${ }^{\text {a }} \mathrm{P}<0.01$; Compared with the model group, ${ }^{b} \mathrm{P}<0.05$; Compared with the western medicine group, ${ }^{\mathrm{c}} \mathrm{P}<0.05$

\subsection{Effect of HJJR on Apoptosis of Aortic Cells}

The results of aortic apoptosis test showed that the positive staining were brown and yellow, located in the nucleus, and its volume were equal to or smaller than that of normal nucleus (normal nuclei are blue). The results showed that a large number of apoptotic cells appeared in the model group. Compared with the normal group, the cell apoptotic index
(CAI) of the model group were significantly higher $(\mathrm{P}<0.01)$. Compared with the model group, the CAI of administration groups were significantly lower $(\mathrm{P}<0.05)$. HJJR2 had more significant effect than western medicine group $(\mathrm{P}<0.05)$. The HJJR effect were better with increasing dose, but no significant difference between HJJR groups. The results are shown in Figure 1 and Table 2. 


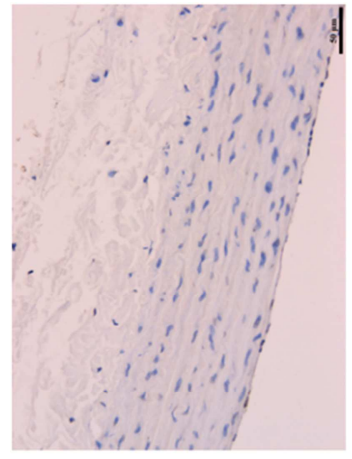

normal group

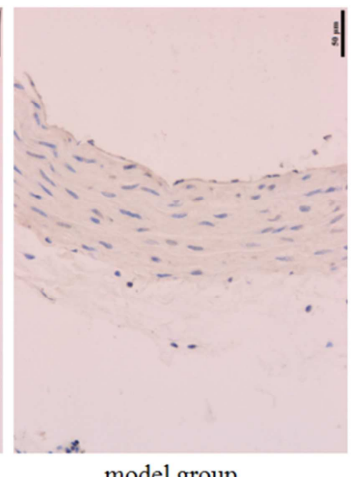

model group

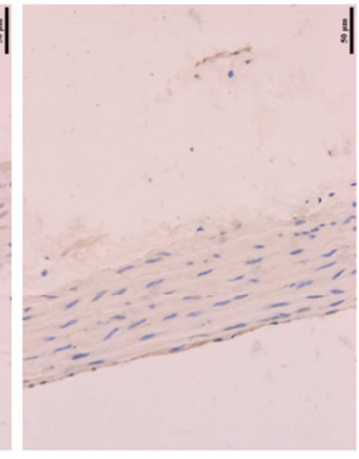

$\mathrm{HJJR}_{1}$ group

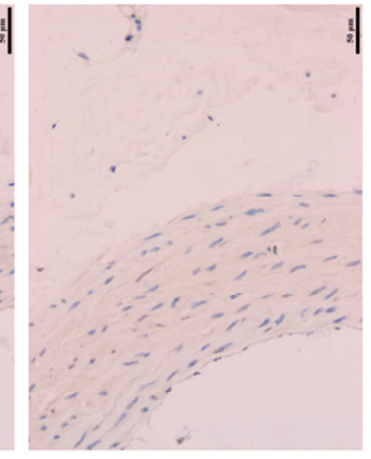

$\mathrm{HJJR}_{2}$ group

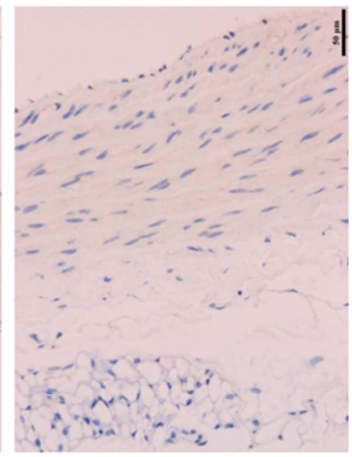

Western medicine group

Figure 1. Aortic cell apoptosis pictures $(\times 200)$.

\subsection{Effect of HJJR on the Expression of TNF-a and IL-6 in Rat Aorta}

Normally, the TNF- $\alpha$ and IL-6 in aorta were low expression, which positive expression were brown yellow. Compared with the normal group, the positive protein in the model group were highly stained $(\mathrm{P}<0.01)$. Compared with the model group, the expression of TNF- $\alpha$ and IL- 6 decreased significantly in all administration groups $(\mathrm{P}<0.05)$. Compared with western medicine group, the expression of TNF- $\alpha$ and IL-6 in HJJF2 group decreased $(\mathrm{P}<0.05)$. The positive expression decreased with the increase of HJJF dosage, and the therapeutic effect was dose-dependent, but there w no significant difference between HJJF groups $(\mathrm{P}>0.05)$. The results are shown in Figures 2, 3 and Table 2.

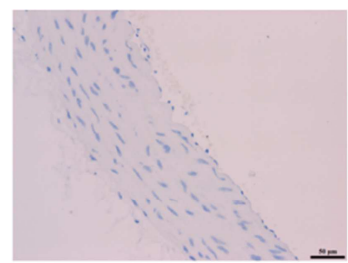

normal group

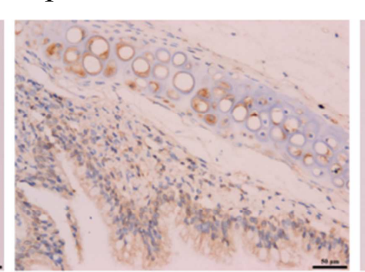

model group $\times 200$

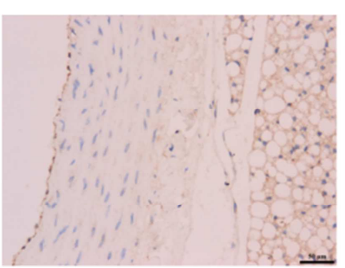

Western medicine group

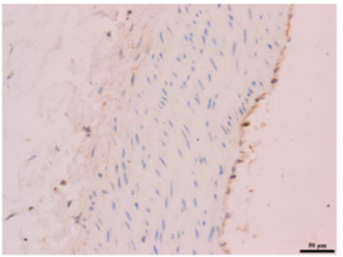

$\mathrm{HJJR}_{1}$ group

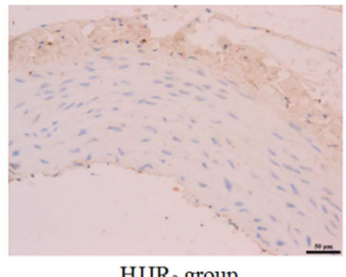

$\mathrm{HJJR}_{2}$ group

Figure 2. The expression of TNF- $\alpha$ in aorta $(\times 200)$.

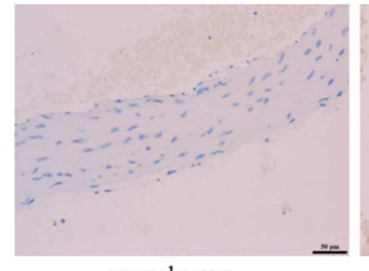

normal group

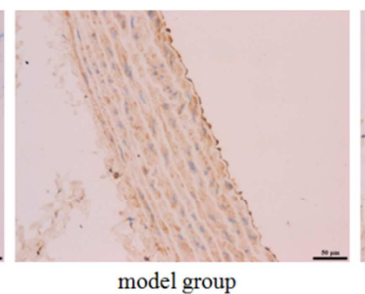

model group

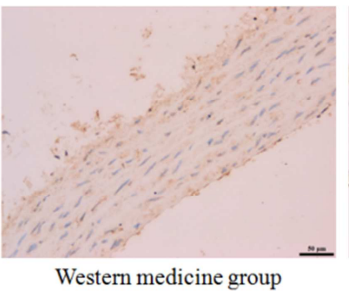

Western medicine group

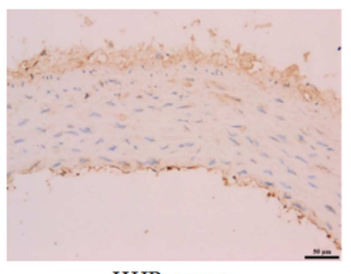

HJJR $_{1}$ group

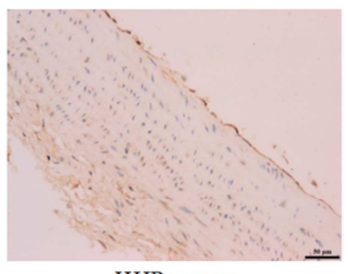

$\mathrm{HJJR}_{2}$ group

Figure 3. The expression of IL-6 in aorta $(\times 200)$.

Table 2. Effect of HJJR on the expression of TNF- $\alpha$ and IL-6 in aorta $(\bar{x} \pm s)$.

\begin{tabular}{|c|c|c|c|c|}
\hline Group & $\mathbf{n}$ & CAI (\%) & TNF- $\alpha$ expression (\%) & IL-6 expression (\%) \\
\hline normal group & 25 & $2.5 \pm 0.27$ & $1.69 \pm 0.29$ & $1.89 \pm 0.31$ \\
\hline model group & 25 & $41.6 \pm 0.55^{\mathrm{a}}$ & $19.6 \pm 6.15^{\mathrm{a}}$ & $21.7 \pm 6.85^{\mathrm{a}}$ \\
\hline $\mathrm{HJJR}_{1}$ group & 26 & $31.1 \pm 0.87^{\mathrm{ab}}$ & $8.48 \pm 3.11^{\mathrm{ab}}$ & $9.79 \pm 4.15^{\mathrm{ab}}$ \\
\hline $\mathrm{HJJR}_{2}$ group & 25 & $29.3 \pm 0.49^{\mathrm{abc}}$ & $6.27 \pm 2.96^{\mathrm{abc}}$ & $8.11 \pm 3.88^{\mathrm{abc}}$ \\
\hline western medicine group & 26 & $33.4 \pm 0.62^{\mathrm{ab}}$ & $9.15 \pm 2.57^{\mathrm{ab}}$ & $11.67 \pm 2.11^{\mathrm{ab}}$ \\
\hline
\end{tabular}

Notes: Compared with the normal group, ${ }^{\text {a }} \mathrm{P}<0.01$; Compared with the model group, ${ }^{\mathrm{b}} \mathrm{P}<0.05$; Compared with the western medicine group, ${ }^{\mathrm{c}} \mathrm{P}<0.05$

\subsection{Effects of HJJR on Transcriptional Levels of GRP78 and CHOP in Rat Aorta}

Compared with the normal control group, the transcription of GRP78 and CHOP in the model group were significantly increased $(\mathrm{P}<0.01)$. Compared with the model group, the transcription of GRP78 and CHOP mRNA in each administration groups were decreased $(\mathrm{P}<0.05)$. Compared with western medicine group, GRP78 and CHOP mRNA in HJJR2 group decreased more significantly $(\mathrm{P}<0.05)$. The effect of HJJR were better with increasing dose, and the effect were dose dependent, but there were no significant difference between HJJR groups $(\mathrm{P}>0.05)$. The results are shown in Figure 4 and Table 3. 

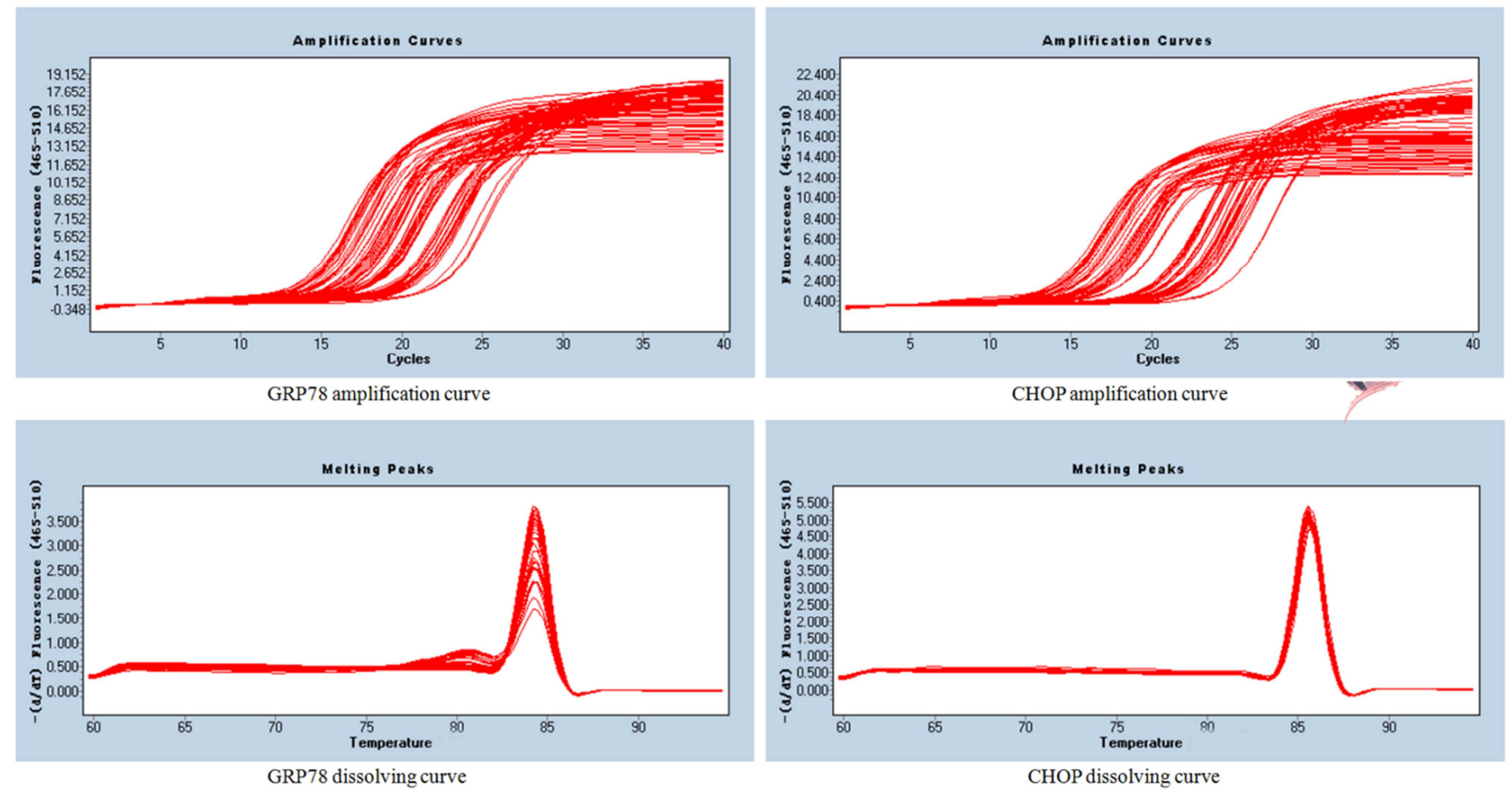

Figure 4. Amplification and dissolution curves of GRP78 and JNK $m R N A$ in aorta.

Table 3. Comparison of relative transcriptional levels of GRP78 and CHOP $m R N A$ in rats aorta $\left(2^{-\Delta \Delta^{C t}}, \bar{x} \pm S\right)$.

\begin{tabular}{llll}
\hline Group & n & GRP78 & CHOP \\
\hline normal group & 25 & $1.07 \pm 0.23$ & $1.06 \pm 0.27$ \\
model group & 25 & $7.11 \pm 0.37^{\mathrm{a}}$ & $7.75 \pm 0.25^{\mathrm{a}}$ \\
$\mathrm{HJJR}_{1}$ group & 26 & $4.21 \pm 0.87^{\mathrm{ab}}$ & $4.76 \pm 0.93^{\mathrm{ab}}$ \\
$\mathrm{HJJR}_{2}$ group & 25 & $3.24 \pm 0.41^{\mathrm{abc}}$ & $3.20 \pm 0.33^{\mathrm{abc}}$ \\
western medicine group & 26 & $4.31 \pm 0.42^{\mathrm{ab}}$ & $4.91 \pm 0.43^{\mathrm{ab}}$ \\
\hline
\end{tabular}

Notes: Compared with the normal group, ${ }^{\mathrm{a}} \mathrm{P}<0.01$; Compared with the model group, ${ }^{\mathrm{b}} \mathrm{P}<0.05$; Compared with the western medicine group, ${ }^{\mathrm{c}} \mathrm{P}<0.05$

\section{Discussion}

Endoplasmic reticulum is an important organelle in eukaryotic cells that regulates protein synthesis, folding, glycosylation modification and translocation. It is also the site for steroid, cholesterol and lipid synthesis. Under pathological conditions, inflammation induced by hypoxia, hyperglycemia and oxidative stress can lead to accumulation of unfolded or misfolded proteins in the endoplasmic reticulum, which will trigger a series of intracellular stress responses, called endoplasmic reticulum stress (ERS) [7]. Glucose regulated protein 78 (GRP78) is the feeling molecular for ER homeostasis. When severe ERS sustained, GRP78 will dissociate with protein kinase $\mathrm{R}$ like endoplasmic reticulum kinase (PERK), activating transcription factor 6 (ATF6) and inositol demand enzyme 1 (IRE-1) three molecules, which then activate the downstream three apoptotic signaling pathways, CCAAT enhancer binding protein homologous protein (CHOP), cysteine-containing aspartate specific protease-12 (caspase-12), c-JUN terminal kinase (JNK), leading to apoptosis [8]. CHOP is a stress-specific transcription factor of endoplasmic reticulum, which is mainly induced by ERS activating PERK pathway. when ERS continued and the stress intensity increased, PERK induce CHOP expressing, which in turn activate the expression of the growth arrest and DNA damage-inducing genes 34 (GADD34), endoplasmic reticulum redox factor 1 (ERO1), death receptor (DRS) [9]. GADD34 can promote the dephosphorylation of eukaryotic translation initiation factor $2 \alpha$ (Eif $2 \alpha$ ), thereby promoting the synthesis of ERS apoptotic pathway-related proteins; ERO1 can encode an ERS oxidase to make endoplasmic reticulum peroxidized; DRS is a cell surface death receptor, which can activate caspase cascade reaction and induce apoptosis. In addition, CHOP can inhibit the expression of anti-apoptotic gene BCL-2, deplete glutathione, induce the production of oxygen free radicals and promote cell apoptosis [9].

Chronic inflammation mediated by macrophages and $\mathrm{T}$ lymphocyte exists during the development of AS, accompanied by unbalanced proliferation and migration of smooth muscle cells and formation of atherosclerotic plaques [10]. Diabetic macroangiopathy is one of the chronic complications of diabetes mellitus, which may cause type 2 diabetic patients disability and death, and whose main pathological feature is atherosclerosis (AS) [11]. Diabetes mellitus results in impairment of the normal pathway of glucose metabolism, over-activation of four bypass pathways, including polyol, protein kinase $\mathrm{C}$ (PKC), advanced glycation end products (AGEs) and hexosamine pathways, enhances the activity of reduced coenzyme II (NADPH), and produces excessive reactive oxygen species (ROS) in cells, hinders the folding of protein's spatial structure, leads to the accumulation of unfolded protein, and then induces ERS, affecting the endoplasmic reticulum homeostasis [12]. At the same time, ROS produced by oxidative stress can oxidize low density 
lipoprotein (LDL) to oxidized low density lipoprotein (ox-LDL). Moreover, endothelial cells, smooth muscle cells and mononuclear macrophages in the arterial wall can also oxidize LDL to form ox-LDL, which is highly affinity to macrophage scavenger receptor, with no negative feedback mechanism, resulting in the accumulation of cholesteryl esters in macrophages to form foam cells [13-14]. In addition, the accumulation of excessive metabolites such as cholesterol, free fatty acids, AGEs, homocysteine in smooth muscle cells and monocyte macrophages caused by diabetes mellitus can persistently over-activate the unfolded protein response, leading to the activation of IRE1, PERK and ATF6 signaling pathways [15]. On the one hand, when IRE1 is activated, it binds with TRAF2 to form IRE1 $\alpha$-TRAF2 complex, which can recruit IkB kinase, phosphorylate and degrade $\mathrm{IkB}$, release $\mathrm{NF}-\mathrm{kB}$ into nucleus and activate the transcription of pro-inflammatory genes. In addition, IRE1-TRAF2 complex can recruit and activate protein kinase $\mathrm{JNK}$, then phosphorylate and activate transcription activating protein-1 (AP-1) to promote the expression of pro-inflammatory genes [17], inducing the expression of VCAM-1 and MCP-1, promoting monocytes to enter atherosclerosis, inducing $\mathrm{T}$ cells and macrophages to aggregate in atherosclerosis, then promote the development of atherosclerosis [18]. On the other hand, the activation of PERK can activate downstream CHOP apoptotic signal, integrate and amplify CHOP transcription and induce apoptosis [9]. Persistent inflammation and cell apoptosis in the arteries increase plaque instability, promote thrombosis and trigger acute cardiovascular events [16]. The expression of endoplasmic reticulum molecular chaperone was found in the samples obtained by percutaneous transluminal plaque resection, and the apoptotic pathway of CH0P was activated in unstable plaques [19]. The apoptosis of smooth muscle cells and macrophages resulted in the accumulation of cell debris in the vascular wall, which could enhance the vulnerability of atherosclerotic plaques.

Diabetes mellitus belongs to the category of Xiao Ke in TCM. At the beginning, its pathological features are "Yin deficiency, dryness and heat", and For a long time, it gradually develop "Qi-Yin deficiency and dry-heat". Qi deficiency leads to blood stagnation due to inability to transport blood; Qi deficiency also leads to phlegm accumulation due to unable defuse dampness, leading to stagnation of blood stasis and phlegm turbidity, and throttling in the veins to accumulate (AS) [20]. Therefore, the etiology and pathogenesis of diabetic atherosclerotic plaque is similar to that of "accumulated disease" in TCM, and resemble to Ming Dynast «Jingyue Quanshu-accumulation» accumulated diseases "The tangible illness all are dietary stagnation, or pus and blood retention, all are accumulation disease". Therefore, "deficiency of both Qi and Yin, accumulation of blood stasis and toxin" are the basic pathogenesis of diabetes mellitus with atherosclerotic plaque formation. The treatment should, on the basis of "nourishing Yin, invigorating Qi, activating blood circulation and resolving phlegm", attach importance to the therapeutic effect of "Softening hardness, dissipating stasis and eliminating accumulation”, just as Qing Dynasty doctor Yu Jiayan`s «The
Law of Medical Doors» discussion on chest pain syndrome, "Qi resistance should be unobstructed, blood stasis be dispersed, depression be opened, and fire stagnation be dissipated or eliminated", in which, the treatments of "dispersed" and "dissipated" were mentioned. The treatment principle of "invigorating Qi and nourishing Yin, activating blood circulation and detoxifying, softening firmness and eliminating accumulation"for diabetic AS and the "treating accumulated diseases" are homologous in TCM "treating different diseases with the same treatment". HJJR consists of ginseng, Ophiopogon japonicus, Schisandra chinensis, Astragalus membranaceus, Rehmannia glutinosa, Yam, Cornus officinalis, Salvia miltiorrhiza, Rhubarb, Coptis chinensis, turtle shell, peach kernel and peony bark. Among them, Ginseng, Ophiopogon japonicus and Schisandra chinensis are Li Xiao (one of the four great doctors in Jin-Yuan Dynasty) famous prescriptions for nourishing Yin and tonfying Qi. Rehmannia glutinosa, yam and cornel are Ming Dynasty physician Zhang Jingyue's “Zuogui Pill”for treating deficiency of Yin. Turtle shell, rhubarb, peach kernel and Danpi are Eastern Han Dynasty famous doctor Zhang Zhongjing's "Turtle shell pills" ingredients, with functions of "activating blood circulation, detoxifying and softening hardness". In the prescription, Astragalus membranaceus is added to strengthen Qi-invigorating and detoxification, Coptis chinensis to strengthen heat-clearing, Dampness-drying and detoxification, and Kadan to strengthen blood-activating and stasis-dispersing. It is an innovation in dealing with diabetic AS to seek the treatment ideas from the compatibility of tonifying Qi, nourishing Yin, activating blood circulation and removing blood stasis, clearing away heat and detoxification, and softening firmness and dispersing knots. Clinical studies have found that HJJR is effective in diabetic patients complicated with acute coronary syndrome (patients without revascularization or patients after $\mathrm{PCI}$ ). compared with routine western medicine treatment, HJJR significantly reduced the symptoms of chest tightness and chest pain, total score of syndromes, ST segment depression leads, T wave low flat leads and $T$ wave inverted leads in patients with non-revascularization, and could regulate immune disorders, down-regulate proinflammatory factors CRP, IL-6 and TNF- $\alpha$, and increase the levels of anti-inflammatory factors IL-10 and adiponectin (ADPN) [21]. In patients after PCI, HJJR can significantly reduce the clinical symptoms of palpitation, chest tightness, fatigue and shortness of breath, compared with conventional western medicine. It can also improve cardiac remodeling, enhance cardiac function and intervene the occurrence and development of diabetic coronary heart disease after PCI [22].

\section{Conclusion}

In diabetes mellitus, impaired normal pathways of glucose metabolism, overactivation of bypass pathways, excessive reactive oxygen species (ROS) production in cells, oxidative stress, and lipid metabolism disorders caused by insulin resistance, all affect the homeostasis of endoplasmic reticulum, 
induce ERS and persistent over-activation of unfolded protein response, which, on the one hand, activate the transcription of pro-inflammatory genes and induce vascular inflammation, and on the other hand, sensitize downstream CHOP apoptotic signals, integrate and amplify CHOP transcription, and induce apoptosis. Persistent inflammation and apoptosis will increase the development of atherosclerosis. This study showed that after successful diabetes model establishment, insulin resistance was obvious, fasting insulin level was decreased, insulin sensitivity was decreased, and GRP78 protein was abundantly expressed, which indicated that GRP78 was a switch molecule upstream of ERS signal, and ERS occurred in aortic cells. After drug intervention, insulin sensitivity was increased, insulin resistance was improved, $\mathrm{GHb}$ and IRI were decreased, TNF- $\alpha$ and IL- 6 levels were decreased, GRP78 and CHOP mRNA were down-regulated, and aortic cell apoptosis was reduced, and the effect of HJJR group was more significant with the increase of dose. The therapeutic effect of HJJR lies in correcting insulin resistance, alleviating the coupling reaction of oxidative stress-ERS-cell apoptosis in diabetes mellitus, inhibiting the ERS apoptosis through CHOP bypass pathway, reducing aorta cells apoptosis, alleviating inflammation of aortic intima, thus stabilizing atherosclerotic plaques and protecting arteries, playing multi-link, multi-channel and multi-target role of "nourishing Yin and invigorating Qi, promoting blood circulation and detoxifying" overall TCM regulation.

\section{Acknowledgements}

This research is supported by National Natural Science Foundation of China (81460698,81960833); Guangxi emphasis research and development program (2017AB45042); Guangxi Natural Science Foundation (2015GXNSFAA139221).

\section{References}

[1] CHEN Yuanyuan, CHEN Yafen, SUN Xueran, et al. Regulation mechanism of AGEs on FSTL1 expression in diabetes with atherosclerosis. International Journal of Cardiovascular Disease, 2019, 46 (1): 31-35.

[2] ZHONG Qiaoqing, ZHU Lingyan, ZHANG Guogang, et al. Atherosclerotic cardiovascular disease in diabetes--mechanisms, clinical represent and management. Chinese Journal of Cardiovascular Review, 2018, 16 (12): 1069-1072.

[3] Fu Xianzhao, Huang Zhenfeng, Huang Wenhua, et al. Review and Prospect of the Preventive Effect of "Supplementing Qi and Nourishing Yin, Activating Blood Circulation and Detoxifying" on Diabetes Mellitus Complicated with Acute Coronary Syndrome. American Journal of Clinical and Experimental Medicine. 2018, 6 (4): 87-93.

[4] Fu Xianzhao, Huang Wenhua, Li Chunyan, et al. Effect of Huoxue Jiedu Jiangtang Formulation on Endoplasmic Reticulum Stress in Diabetic Atherosclerosis. American Journal of Clinical and Experimental Medicine. 2018, 6 (5): $118-124$.
[5] Jing G, Xing-zhi W, Tao W, et al. Molecular signal networks and regulating mechanisms of the unfolded protein response. Biomed \& Biotechnol, 2017, 18 (1): 1-14.

[6] GUAN Li Ying, XU CaiMin, PAN Hua Zhen. Endoplasmic Reticulum Stress-induced Apoptosis. Progress in Biochemistry and Biophysics, 2007, 34 (11): 1136-1141.

[7] Maamoun H, Abdelsalam SS, Zeidan A, et al. Endoplasmic Reticulum Stress: A Critical Molecular Driver of Endothelial Dysfunction and Cardiovascular Disturbances Associated with Diabetes. Int J Mol Sci. 2019 Apr 3; 20 (7). pii: E1658.

[8] Feng SQ, Zong SY, Liu JX, et al. VEGF antagonism attenuates cerebral ischemia/reperfusion- induced injury via inhibiting endoplasmic reticulum stress-mediated apoptosis. Biol Pharm Bull. 2019 Mar 2. doi: 10.1248/bpb.b18-00628

[9] Chen Y, Zhang B, Cao S, et al. GanDouLing combined with Penicillamine improves cerebrovascular injury via PERK/eIF $2 \alpha / \mathrm{CHOP}$ endoplasmic reticulum stress pathway in the mouse model of Wilson's disease. Biosci Rep. 2018 Sep 19; 38 (5). pii: BSR20180800.

[10] TIAN JinFan, LIU Yue, LI Li Zhi, et al. reticulum stress, autophagy and their crosstalk: the potential treatment targets for atherosclerosis. Chinese Bulletin of Life Sciences, 2017, 29 (4): 356-362.

[11] ZHANG Wen. Research progress of the relevance between endoplasmic reticulum stress and diabetes associated atherosclerosis. Clinical journal of traditional Chinese medicine, 2018, 27 (11): 1620-1622.

[12] Gong J, Wang XZ, Wang T, et al. Molecular signal networks and regulating mechanisms of the unfolded protein response. J Zhejiang Univ Sci B, 2017, 18 (1): 1-14.

[13] Zhang K, Kaufman RJ. From endoplasmic-retieulum stress to the inflammatory response. Nature, 2008, 454: 455-462.

[14] Seimon T, Tabas I. Mechanisms and consequences of macrophage apoptosis in atherosclerosis [J]. J Lipid Res, 2009, 50: S382-S387.

[15] Jia LX, Zhang WM, Li TT, et al. ER stress dependent microparticles derived from smooth muscle cells promote endothelial dysfunction during thoracic aortic aneurysm and dissection. Clin Sci (Lond). 2017 Jun 7; 131 (12): 1287-1299.

[16] $\mathrm{Hu} \mathrm{H}$, Wang $\mathrm{C}$, Jin $\mathrm{Y}$, et al. Catalpol Inhibits Homocysteine-induced Oxidation and Inflammation via Inhibiting Nox4/NF- $\mathrm{BB}$ and GRP78/PERK Pathways in Human Aorta Endothelial Cells. Inflammation. 2019 Feb; 42 (1): 64-80. doi: 10.1007/s10753-018-0873-9.

[17] Tabas I, Seimon T, Timmins J, et al. Macrophage apoptosis in advanced atherosclerosis. Ann N Y Acad Sci. 2009, 1173 (Suppl 1): E40-45.

[18] Yuan J, Chen M, Xu Q, et al. Effect of the Diabetic Environment On the Expression of miRNAs in Endothelial Cells: Mir-149-5p Restoration Ameliorates the High Glucose-Induced Expression of TNF- $\alpha$ and ER Stress Markers. Cell Physiol Biochem, 2017, 43 (1): 120-135.

[19] Lim W, Timmins J, Seimon T, et al. Signal transducer and activator of transcription-1 is critical for apoptosis in macrophages subjected to endoplasmic reticulum stress in vitro and in advanced atherosclerotic lesions in vivo [J]. Circulation, 2008. 117 (7): 940-951. 
[20] FU Xian-zhao, XU Jing, HUANG WenHua, et al. Strategy of Stasis-resolving and Detoxification on Diabetic Coronary Heart Disease [J]. JETCM, 2014, 23 (11): 2024-2027.

[21] FU Xian-zhao, XU Jing, LI ChunYan, et al. Effects of Huoxue Jiedu Jiangtang formulation on the balance between pro-and anti-inflammatory cytokines in non-revascularization patients with diabetic acute coronary syndrome $[\mathrm{J}]$. Chin J TCM WM Crit Care, 2016, 23 (1): 6-10.

[22] FU Xian-zhao, XU Jing, LI ChunYan, et al. Effect of Huoxue Jiedu Jiangtang formulation on heart remodeling in diabetic patients with acute coronary syndrome after PCI [J]. Chin J TCM WM Crit Care, 2016, 23 (2): 117-121. 